\title{
Doença de Creutzfeldt-Jakob: Apresentação Atípica de uma Doença Muito Rara
}

\section{Creutzfeldt-Jakob Disease: Atypical Presentation of a Very Rare Disease}

\author{
Renato OLIVEIRA $\triangle^{1,2}$, Marta DIAS ${ }^{3}$, Inês Brás MARQUES ${ }^{1}$ \\ Acta Med Port 2021 Sep;34(9):619-623 - https://doi.org/10.20344/amp.13117
}

\section{RESUMO}

A doença de Creutzfeldt-Jakob, manifesta-se habitualmente como demência rapidamente progressiva. Apresentamos um caso de um doente de 59 anos com quadro súbito de parésia facial central e disartria, seguindo-se mioclonias no hemicorpo esquerdo. A ressonância magnética crânio-encefálica inicial mostrava hipersinal T2 e difusão no caudado e putamen direitos e líquido cérebro-raquidiano com hiperproteinorraquia. A investigação para causas autoimunes, infecciosas e paraneoplásicas foi negativa. Verificou-se um agravamento progressivo nos meses seguintes para hemiplegia esquerda, disfagia, incontinência urinaria e posterior mutismo acinético. A ressonância magnética crânio-encefálica mostrou evolução para restrição à difusão dos gânglios da base bilateralmente e múltiplas áreas corticais; A eletroencefalografia mostrou atividade periódica e proteína Tau no líquido cérebro-raquidiano elevada. A análise genética revelou mutação c.598G > A. O falecimento ocorreu após quatro meses de doença. Reportamos um caso de doença de Creutzfeldt-Jakob familiar associada a mutação da proteína priónica, com apresentação clínica e radiológica atípicas, nomeadamente sinais focais com instalação súbita, ausência de defeito cognitivo significativo e alterações imagiológicas unilaterais. Na evolução, a clínica e imagem tornaram-se características, permitindo o diagnóstico.

Palavras-chave: Mutação/genética; Priões/genética; Síndrome de Creutzfeldt-Jakob/diagnóstico; Síndrome de Creutzfeldt-Jakob/ genética

\section{ABSTRACT}

Creutzfeldt-Jakob disease typically presents as rapidly progressive dementia. We describe the case of a 59-year-old male patient presenting with sudden onset of central facial palsy and dysarthria, followed by myoclonus of his left upper and lower limbs. Initial brain magnetic resonance showed hyperintensity of the right caudate and putamen on diffusion-weighted imaging and T2 sequences. Cerebrospinal fluid analysis showed increased protein count. The workup to investigate autoimmune, infectious and paraneoplastic causes was negative. Symptoms progressively worsened, with left hemiplegia, dysphagia, urinary incontinence, and, later, akinetic mutism. The follow-up brain magnetic resonance scan revealed hyperintensity of bilateral basal ganglia as well as cerebral cortical abnormalities on diffusion-weighted imaging. Electroencephalography showed periodic activity and tau protein levels in the cerebrospinal fluid were elevated. Genetic analysis showed mutation c-598G > A. The patient died four months later. We report a case of familial Creutzfeldt-Jakob disease with atypical clinical and radiological features, namely neurological focal signs with sudden onset, absence of significant cognitive impairment and unilateral radiological findings. With disease progression, characteristic clinical and radiological features led to the diagnosis.

Keywords: Creutzfeldt-Jakob Syndrome/diagnosis; Creutzfeldt-Jakob Syndrome/genetics; Mutation/genetics; Prions/genetics

\section{INTRODUÇÃO}

As doenças priónicas, ou encefalopatias espongiformes transmissíveis, são doenças degenerativas que afetam o sistema nervoso central de animais e humanos ${ }^{1}$ (Tabela 1). A proteína priónica normal (PrPC), presente em todas as espécies mamíferas, é codificada pelo gene priónico (PRNP) no cromossoma 20,1-3 desconhecendo-se o seu papel fisiológico. Nestas doenças a proteína priónica é convertida numa forma anormal denominada prião. ${ }^{1-3}$

A doença de Creutzfeldt-Jakob (DCJ),forma mais comum de doença priónica humana, é muito rara, com taxa de incidência anual de 1 - 2 casos/milhão a nível global. ${ }^{3}$ A forma esporádica (sDCJ) corresponde a $80 \%$ - 95\% dos casos. ${ }^{2}$ Estima-se que $10 \%$ a $15 \%$ dos casos estejam associados a mutações de PRNP (DCJ familiar/hereditária) e que $1 \%$ sejam iatrogénicos, e relacionados com transplantes de dura-máter ou córnea, e com o uso de instrumentos neurocirúrgicos contaminados. ${ }^{2}$ Existe ainda a forma variante relacionada com o consumo de produtos contaminados, atualmente rara. ${ }^{2}$

A sDCJ tem pico de incidência na sétima década de vida, e caracteriza-se por alteração cognitiva rapidamente progressiva associada a sinais neurológicos, mais frequentemente mioclonias e disfunção cerebelosa, ${ }^{4}$ com sobrevida média de seis meses. ${ }^{2} \mathrm{O}$ diagnóstico definitivo consiste na identificação de priões em tecido cerebral post mortem. Contudo, é possível fazer o diagnóstico utilizando critérios do Centers for Disease Control and Prevention (CDC), que consideram como critérios alterações no eletroencefalograma (EEG), ressonância magnética crânio-encefálica (RM-CE), e marcadores no líquido cefalorraquidiano (LCR) (Fig. 1).

A DCJ familiar é semelhante à forma esporádica, apre-

1. Neurology Department. Hospital da Luz. Lisboa. Portugal.

2. Comprehensive Health Research Centre. Universidade NOVA de Lisboa. Lisboa. Portugal.

3. Neurology Department. Hospital de Santarém. Santarém. Portugal.

$\triangle$ Autor correspondente: Renato Oliveira. renato.silva.oliveira@hospitaldaluz.pt

Recebido: 12 de novembro de 2019 - Aceite: 27 de fevereiro de 2020 - First published: 28 de setembro de 2020 - Online issue published: 01 de setembro de 2021 Copyright $\odot$ Ordem dos Médicos 2021 
Tabela 1 - Encefalopatias espongiformes transmissíveis - Doenças Priónicas Humanas e seus acrónimos, e correspondentes doenças priónicas animais.

\begin{tabular}{cc}
\hline $\begin{array}{c}\text { Doenças humanas priónicas } \\
\text { DCJ esporádica } \\
\text { DCJ iatrogénica } \\
\text { DCJ familiar ou genética } \\
\text { DCJ variante }\end{array}$ & $\begin{array}{c}\text { Encefalopatias espongiformes transmissíveis animais } \\
\text { Tremor epizoótico atípico }\end{array}$ \\
$\begin{array}{c}\text { Síndrome de Gerstmann-Straussler-Scheinker } \\
\text { Insónia fatal esporádica } \\
\text { Insónia fatal familiar }\end{array}$ & $\begin{array}{c}\text { Encefalopatia espongiforme dos visons } \\
\text { Doença crónica emaciante }\end{array}$ \\
$\begin{array}{c}\text { Prionopatia variavelmente sensível à protease } \\
\text { Kngiopatia amilóide cerebral de proteína priónica }\end{array}$ & $\begin{array}{c}\text { Encefalopatia espongiforme bovina } \\
\text { Encefalopatia espongiforme dos felinos }\end{array}$
\end{tabular}

DCJ; doença de Creutzfeldt-Jakob

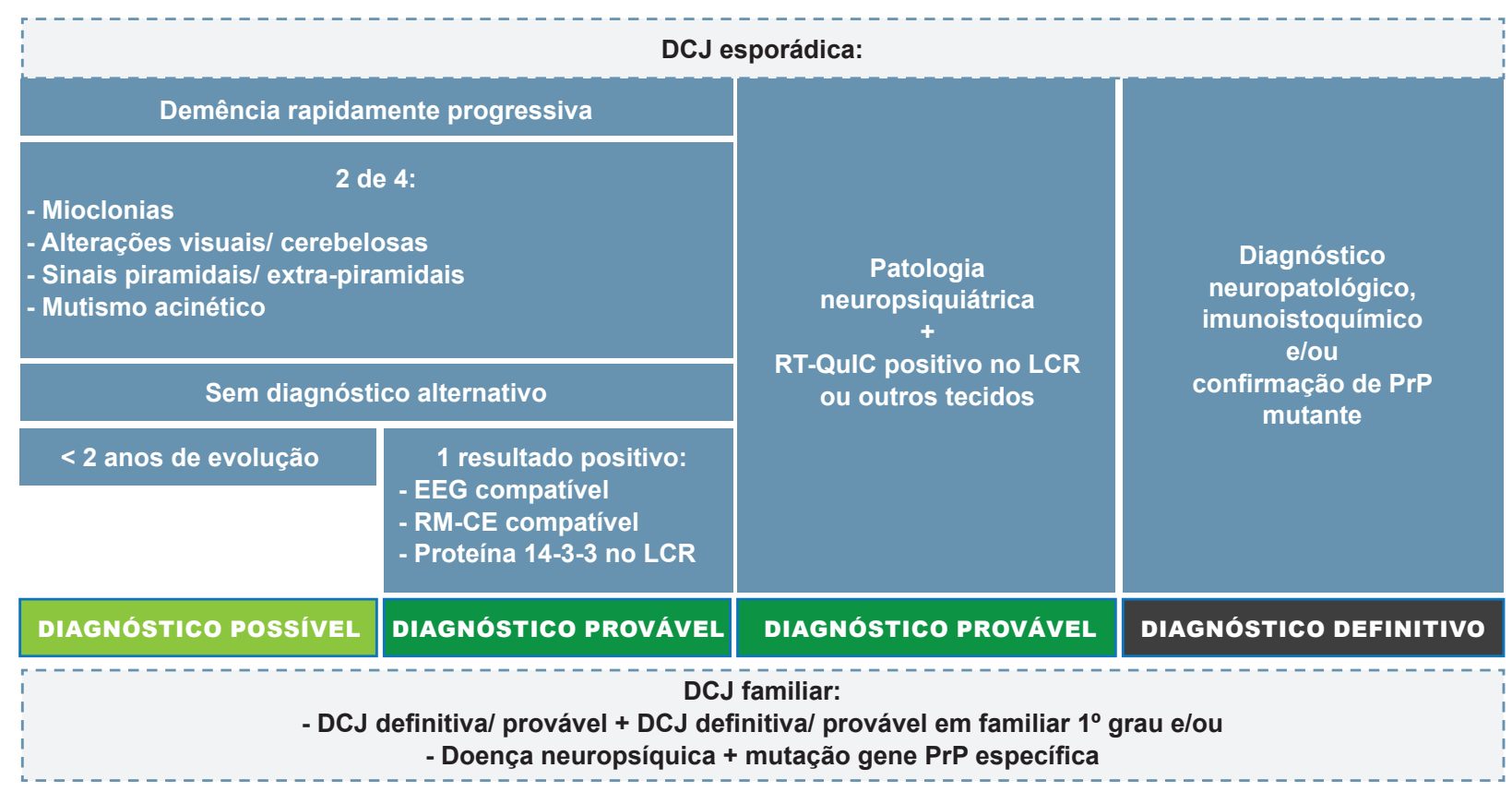

Figura 1 - Critérios do Centers for Disease Control and Prevention para o diagnóstico de doença de Creutzfeldt-Jakob (DCJ) DCJ: doença de Creutzfeldt-Jakob; EEG: eletroencefalograma; LCR: líquido cérebro-raquidiano; PrP: proteína priónica; RM-CE: ressonância magnética crânio-encefálica; RT-QuIC: real-time quaking-induced conversion

sentando-se em idades mais precoces (40 - 50 anos) e com sobrevida ligeiramente maior (até dois anos). ${ }^{2} \mathrm{~A}$ mutação E200K é a mais comum a nível global. ${ }^{2}$

Apresentamos um caso de DCJ definitiva em que a marcha diagnóstica foi desafiadora pela apresentação clínica e radiológica atípica.

\section{CASO CLÍNICO}

Doente do sexo masculino de 59 anos com antecedentes de hemocromatose, acidente isquémico transitório e glaucoma. Apresentava história familiar em dois tios paternos de morte aos 60 anos por doença neurológica não especificada.

Apresentou-se com disartria e parésia facial de instalação súbita, com melhoria em poucos dias. Após uma semana iniciou movimentos involuntários do membro superior esquerdo (MSE), associados a disartria, ligeira anomia (di- ficuldade na nomeação de pessoas e objetos) e discalculia (dificuldade no cálculo) Não apresentava febre ou outra sintomatologia. Ao exame neurológico não apresentava alteração significativa das funções nervosas superiores, com disartria ligeira, parésia facial central direita, movimentos mioclónicos do MSE e hemiface esquerda (Tabela 2).

A RM-CE (Fig. 2A) mostrou alteração em T2 e difusão (DWI) no caudado e putamen direitos, sem envolvimento do estriado contralateral. Por manter agravamento, repetiu RM-CE (Fig. 2B) após cinco dias, tendo-se verificado adicional hipersinal em difusão do córtex fronto-parieto-cingular medial. A investigação realizada para identificação de causas autoimunes, infecciosas e paraneoplásica resultou negativa, LCR apresentava aumento da proteína tau e proteína 14.3.3 normal. O EEG revelou atividade lenta parietal direita e frontotemporal bilateral (Tabela 2). Nas duas semanas seguintes, verificou-se agravamento da disartria 
Tabela 2 - Resumo das características clínicas, laboratoriais e radiológicas ao longo do curso da doença

\begin{tabular}{|c|c|c|c|}
\hline Sexo/ Idade & \multicolumn{3}{|c|}{$\hat{0}, 59$ anos } \\
\hline $\begin{array}{l}\text { Tempo de sintomas } \\
\text { até ao diagnóstico }\end{array}$ & \multicolumn{3}{|c|}{1 mês } \\
\hline Clínica inicial/ evolutiva & $\begin{array}{c}\text { Disartria } \\
\text { Parésia facial central direita } \\
\text { Mioclonias* } \\
\text { Discalculia }\end{array}$ & $\begin{array}{c}\text { Hemiplegia esquerda } \\
\text { Disfagia }\end{array}$ & $\begin{array}{l}\text { Incontinência urinaria } \\
\text { Mutismo acinético }\end{array}$ \\
\hline Laboratorialmente & \multicolumn{3}{|c|}{$\begin{array}{c}\text { Proteína Tau > } 1700 \\
\text { Proteína 14.3.3 negativa }\end{array}$} \\
\hline Diagnóstico diferencial & \multicolumn{3}{|c|}{$\begin{array}{l}\text { Estudo negativo para causas autoimunes, infeciosas, paraneoplásicas, incluindo: } \\
\text { - VS, PCR, autoimunidade sistémica (ANA, SSA/B, ANCAs, SAF, anti-Tiroideus), Ar } \\
\text { anticorpos associados a encefalites auto-imunes (NMDA, LGI1, AMPA, GABA, CAS } \\
\text { - LCR: bandas oligoclonais, exames culturais, exame citológico, pesquisa de vírus } \\
\text { neurotrópicos; } \\
\text { - TC tóraco-abdomino-pélvica }\end{array}$} \\
\hline $\begin{array}{l}\text { Evolução radiológica } \\
\text { (restrição à difusão - RM-CE) }\end{array}$ & $\begin{array}{l}\text { Núcleo caudado e putamen } \\
\text { direito }\end{array}$ & $\begin{array}{l}\text { Córtex fronto-pareito-cingular } \\
\text { medial e pericentral à direita }\end{array}$ & $\begin{array}{l}\text { Gânglios da base } \\
\text { bilateralmente e múltiplas } \\
\text { áreas corticais }\end{array}$ \\
\hline $\begin{array}{l}\text { Evolução eletroencefalográfica } \\
\text { (EEG) }\end{array}$ & Atividade lenta difusa & \multicolumn{2}{|c|}{ Atividade periódica complexos ponta-onda } \\
\hline Tempo de sintomas até à morte & \multicolumn{3}{|c|}{4 meses } \\
\hline
\end{tabular}

EEG: eletroencefalograma; LCR: líquido-céfalo-raquidiano; RM-CE: ressonância magnética crânio-encefálica; đ: sexo masculino; *Mioclonias: movimentos mioclónicos do membro superior esquerdo desencadeados pela extensão do punho e mioclonias rítmicas da hemiface esquerda com expansão aos músculos da região cervical ipsilateral desencadeados pela contração voluntária dos músculos da hemiface esquerda.

e surgimento de ataxia da marcha. Colocou-se a possibilidade de encefalite autoimune, tendo sido administrada metilprednisolona endovenosa (1 g durante cinco dias), sem melhoria.

Nos meses seguintes a deterioração neurológica manteve-se progressiva, com evolução para anartria (incapacidade de articulação verbal) e disfagia, hemiparesia esquerda, incontinência urinária e agravamento cognitivo com alteração da memória recente, desorientação temporal e disfunção executiva. Foi realizada nova RM-CE (Fig. 2C), que mostrou restrição à difusão dos gânglios da base bilateralmente e múltiplas áreas corticais, predominantemente córtex frontal e parietal. O EEG identificou atividade periódica de complexos ponta-onda generalizadas com reatividade à estimulação. $\mathrm{O}$ doente viria a falecer aos quatro meses de doença.

A análise genética efetuada posteriormente resultou positiva para a mutação c.598G > A (E200K).

\section{DISCUSSÃO}

Na prática clínica, a DCJ surge mais frequentemente no diagnóstico diferencial de demência rapidamente progressiva. Nalguns casos, pode mimetizar outras doenças neurológicas ou psiquiátricas e os exames complementares iniciais podem não apresentar alterações características. Estima-se que muitos doentes já ultrapassaram dois terços do curso da doença aquando do diagnóstico. ${ }^{3}$

Os sintomas cognitivos são a forma mais comum de apresentação, seguidos em igual frequência por sintomas cerebelosos, constitucionais e comportamentais $(20 \%$ cada). ${ }^{2}$ Sintomas de disfunção cortical (por exemplo, afasia, apraxia, neglect) ocorrem precocemente em 15\% dos casos, e em metade dos casos durante a evolução da doença. ${ }^{2}$ O fenótipo clássico caracteriza-se por demência rapidamente progressiva associada a ataxia e mioclonias. No caso que relatamos, apenas as mioclonias fizeram parte da apresentação clínica inicial, que consistiu em sinais neurológicos focais de instalação súbita, sem defeito cognitivo significativo. Estão descritas outras apresentações atípicas, incluindo alien-limb, parésias isoladas de pares cranianos, e variantes clínicas como as variantes visual e cerebelosa. ${ }^{1,2,4}$

Até ao momento existem cerca de 50 mutações patogénicas do gene PRNP, sendo a E200K a mais prevalente. ${ }^{5}$ Embora as alterações dos exames complementares sejam semelhantes à sDCJ, existem algumas diferenças clínicas. Crises convulsivas, cefaleia, neuropatia periférica, parésia da supraversão do olhar e alterações do sono (predominantemente insónia) são mais frequentemente reportadas em doentes com mutação E200K. ${ }^{5,6}$

Em Portugal, a maioria dos casos reportados são de sDCJ. Numa das maiores séries de casos, no norte de Portugal, apenas dois dos 11 doentes incluídos tinham história familiar de demência, e apenas um cumpria critérios de DCJ familiar. Não se identificaram mutações PRNP. ${ }^{7}$

Comparativamente à sDCJ, em que a deteção de proteína 14-3-3 no LCR apresenta alta sensibilidade (90\% $96 \%$ ), a elevação deste e de outros biomarcadores do LCR é menos frequente nas formas de DCJ geneticamente determinadas, ${ }^{2,8}$ o que vai ao encontro do caso clínico repor- 

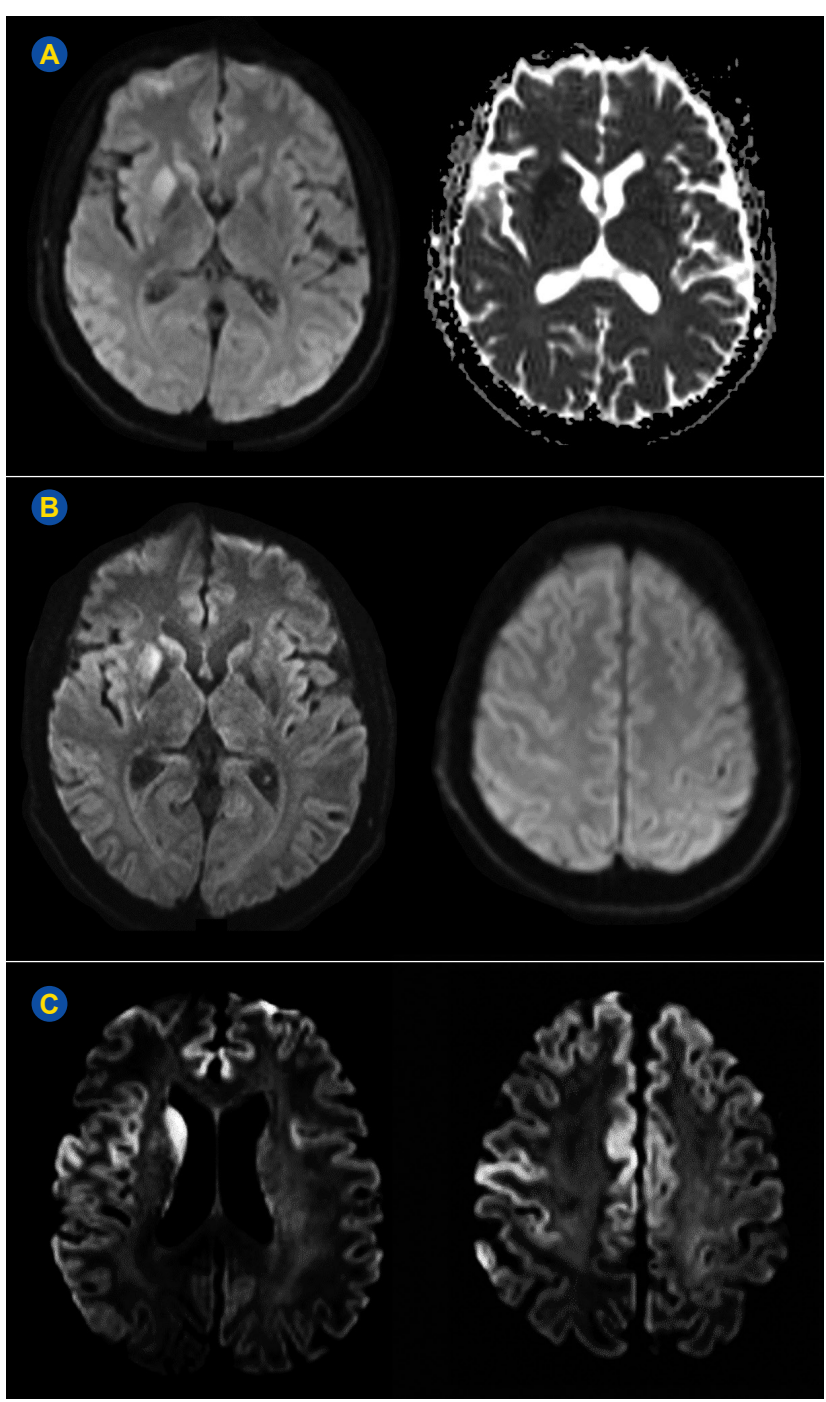

Figura 2 - Evolução radiológica. Evolução das lesões com restrição à difusão na RM-CE ao longo do curso da doença. (A) Alteração em T2 e difusão no caudado e putamen direito. Sem envolvimento do estriado contralateral; (B) Restrição à difusão no caudado e putamen direito, com discreto hipersinal em difusão do córtex fronto-parieto-cingular medial e pericentral a direita. (C) Restrição à difusão dos gânglios da base bilateralmente e múltiplas áreas corticais; RM-CE - ressonância magnética crânio-encefálica.

tado, em que não se detetou proteína 14-3-3 no LCR.

A RM-CE é considerada o exame complementar mais útil na investigação, com sensibilidade global de $92,3 \% .{ }^{9}$ As alterações clássicas incluem hipersinal do caudado, putamen ou córtex (ou combinação entre estes) especialmente

\section{REFERÊNCIAS}

1. Head MW, Ironside JW. Review: Creutzfeldt-Jakob disease: prion protein type, disease phenotype and agent strain: CJD and prion agent strain. Neuropathol Appl Neurobiol. 2012;38:296-310.

2. Geschwind MD. Prion diseases. Continuum. 2015;21:1612-38.

3. Kojima G, Tatsuno BK, Inaba M, Velligas S, Masaki K, Liow KK. Creutzfeldt-Jakob disease: a case report and differential diagnoses. Hawaii J Med Public Health J Asia Pac Med Public Health. 2013;72:1369.

4. Takada LT, Kim MO, Cleveland RW, Wong K, Forner S, Gala I, et al. Genetic prion disease: experience of a rapidly progressive dementia center in the United States and a review of the literature. Am J Med Genet B Neuropsychiatr Genet. 2017;174:36-69. nas sequências de difusão e FLAIR, mas também em T2. No caso apresentado, a RM-CE inicial mostrou hipersinal em T2 e difusão no caudado e putamen apenas à direita. A maioria dos estudos longitudinais mostra que usualmente as alterações do putamen e caudado são bilaterais e simétricas, apesar de inicialmente o hipersinal envolver apenas parte do putamen. Alguns estudos reportam lesões unilaterais em DWI, ${ }^{6,9}$ numa fase precoce da doença, mas são anedóticos os casos com lesões unilaterais em T2. ${ }^{10}$

$\mathrm{Na}$ prática clínica deve ser tida em consideração a possibilidade de apresentações atípicas de DCJ, sendo importante nestes casos a exclusão dos diagnósticos diferenciais. Exames complementares mais sensíveis e específicos, como as recentes técnicas de real-time quaking-induced conversion (RT-QuIC) que permitem detetar quantidades pequenas de proteína priónica no LCR e até no epitélio nasal11,12, podem permitir um diagnóstico confirmatório mais precoce.

\section{CONTRIBUTO DOS AUTORES}

RO: Conceptualização, redação e revisão.

MD: Conceptualização e revisão.

IBM: Redação e revisão.

\section{PROTECÇÃO DE PESSOAS E ANIMAIS}

Os autores declaram que os procedimentos seguidos estavam de acordo com os regulamentos estabelecidos pelos responsáveis da Comissão de Investigação Clínica e Ética e de acordo com a Declaração de Helsínquia da Associação Médica Mundial.

\section{CONFIDENCIALIDADE DOS DADOS}

Os autores declaram ter seguido os protocolos do seu centro de trabalho acerca da publicação de dados.

\section{CONSENTIMENTO INFORMADO}

Obtido.

\section{CONFLITOS DE INTERESSE}

Os autores declaram não ter qualquer conflito de interesse relativamente ao presente artigo.

\section{FONTES DE FINANCIAMENTO}

Não foi utilizada nenhuma bolsa ou subsídio para a realização do trabalho.

5. Gao LP, Shi Q, Xiao K, Wang J, Zhou W, Chen C, et al. The genetic Creutzfeldt-Jakob disease with E200K mutation: analysis of clinical, genetic and laboratory features of 30 Chinese patients. Sci Rep. 2019;9:1836

6. Ukisu R, Kushihashi T, Kitanosono T, Fujisawa H, Takenaka H, Ohgiya $\mathrm{Y}$, et al. Serial diffusion-weighted MRI of Creutzfeldt-Jakob disease. Am J Roentgenol. 2005;184:560-6.

7. Silva AM, Pires MM, Leite AJ, Honavar M, Mendes A, Correia M, et al. Estudo retrospectivo da doença de Creutzfeldt-Jakob diagnosticada no norte de Portugal entre 1993-2002: características demográficas, clínicas e neuropatológicas. Arq Neuropsiquiatr. 2003;61:950-6.

8. Collins S, Boyd A, Fletcher A, Gonzales M, McLean C, Byron K, et 
al. Creutzfeldt-Jakob disease: diagnostic utility of 14-3-3 protein immunodetection in cerebrospinal fluid. J Clin Neurosci. 2000;7:203-8.

9. Collie DA, Sellar RJ, Zeidler M, Colchester AC, Knight R, Will RG. MRI of Creutzfeldt-Jakob disease: imaging features and recommended MRI protocol. Clin Radiol. 2001;56:726-39.

10. Murata T, Shiga Y, Higano S, Takahashi S, Mugikura S. Conspicuity and evolution of lesions in Creutzfeldt-Jakob disease at diffusion-weighted imaging. AJNR Am J Neuroradiol. 2002:23:1164-72.

11. Green AJ. RT-QulC: a new test for sporadic CJD. Pract Neurol. 2019;19:49-55.

12. Orrú CD, Bongianni M, Tonoli G, Ferrari S, Hughson A, Groveman B, et al. A test for Creutzfeldt-Jakob disease using nasal brushings. N Engl $J$ Med. 2014;371:519-29. 